\title{
Study on the Technology of the Coal Mining Safety Monitoring System
}

\author{
Zhi Chang, Zhangeng Sun \& Junbao Gu \\ School of Mechanical and Electronic Engineering, Tianjin Polytechnic University \\ Tianjin 300160, China \\ E-mail: changzhi2007.cool@163.com
}

\begin{abstract}
New development and application of the wireless radio frequency make the application of the RFID (Radio Frequency Identification) technology become more and more extensively, and combining the characteristics of RFID technology with the mine, we established an underground safety monitoring system with integrality, real-time and flexibility, which could automatically alarm when dangers happen and enhance the search and rescue efficiency. The system could manage the harm-gas concentration, planning arrangement of workers, access control for the works passing in and out laneway, distribution of laneway personnel and workers' data, realize the informationization and visualization of the underground management, and enhance the mining production management level and the work safety level of the mine.
\end{abstract}

Keywords: RFID, Safety monitoring system, E-tag, Reader-writer

Mining accidents often happened in China in recent years, and except for mine owners' weak safety and legal consciousness, the lagged safety establishments and the imperfect managements of the mining personnel and equipments were the important causes. Through analyzing several extra serious accidents in the near period, following common problems generally existed (Qiu, 2007, P.28-29), (1) the information communication between the ground personnel and the underground was not timely, (2) ground personnel could not dynamically grasp the underground personnel's distribution and operation situation, and could not exactly position underground personnel, (3) once the mine accident happened, the efficiency of rescue was low, and the effect was worse. Therefore, it is very important and urgent to exactly and quickly carry out the coal mining safety monitoring function (Liu, 2004, P.22-25 \& Guo, 2005), effectively manage the miners, and ensure the high-efficiency operation of the rescue.

The coal mining personnel and vehicle safety monitoring system proposed in the article can track, monitor and position the harmful gases, personnel and vehicles in the mine real time, and offer dynamic information about the mine laneway network, personal position, vehicle position, dangerous region and corresponding clews for ground personnel. If the accident happens, the system also can inquire about the personnel distribution, the personnel quantity, the personnel withdrawal line to offer scientific references for the accident rescue from the monitoring computer. At the same time, the mangers can utilize the daily attendance function in the system to implement attendance management for miners.

\section{Introduction of the RFID technology}

RFID (Zhou, 2006 \& Nadeem Raza, 1999) is a sort of non-contact automatic identification technology, and it can automatically identify the objective by the radio frequency signals, quickly track the goods and exchange data. The identification work needs not human operation, and can work under various severe environments. RFID technology can identify high-speed objects and identify multiple tags simultaneously, and the operation is quick and simple. RFID technology was generated in the Second World War, and it is the successor of the traditional code technology, and its major core part is the E-tag, and the identification system could read the information stored in the E-tag through the radio waves emitted by the reader-writer from a few centimeters to a few meters, and identify the identity of the goods, people and objects represented by the E-tag.

\subsection{Composing of RFID system}

RFID system (seen in Figure 1) is generally composed by E-tag, reader-writer and computer communication network (Simson Garfinekel, 2005).

\subsubsection{E-tag}

E-tag stores correlative information of the identified objects, and it is usually put on the identified objects, and the 
information stored by it can be read and written by the radio frequency reader-writer through the non-contact mode.

\subsubsection{Reader-writer}

The reader-writer is the equipment which can be used to read and write the information of the E-tag by the radio frequency technology. The tag information read by the reader-writer can be managed and transmitted by the computer network system.

\subsubsection{Computer communication network}

In the RFID system, the computer communication network is generally used to manage the data and complete the function of communication transmission. The reader-writer can connect with the computer communication network by the standard interface to realize the communication and data transmission.

\subsection{Work principle of RFID system}

RFID technology is a sort of non-contact information transfer mode realized by the radio frequency signals through space coupling (alternating magnetic field or electromagnetic field), and it identifies the objective through the transferred information. Its work principle can be described as follows (Klaus Finkenzeller, 2003): reader-writer emits energy in one region, form the electromagnetic field, and the size of the region is decided by the work frequency and antenna size, and when the E-tag passes this region, the system checks the signal of the reader-writer and stores the electromagnetic wave energies emitted by the reader-writer to be the electric energy needed by the tag, and the tag transmits the interior information to the reader-writer by the form of the radio wave, and the reader-writer accepts the data of the E-tag, encodes and implement fault check to judge the validity of the data, and transfers the data to the computer communication network for the data processing.

\subsection{Technical characteristics of RFID}

\subsubsection{Data read and write}

RFID reader can read the data to the database without contact, and process multiple tags once, and write the logistic processing state into the tag for the logistic processing in the next stage.

\subsubsection{Miniaturized and diverse form}

RFID will not be limited by the size or form when it reads data, so it needs not to use the paper with fixed size or print quality to fit for the precision. In addition, E-tag of RFID can be applied in different products by small size, so we can more flexibly control the production of the products, especially the application on the production line.

\subsubsection{Anti-pollution}

RFID possesses strong anti-pollution nature for water, oil or drugs. And in the dark or polluted environment, RFID also can read data.

\subsubsection{Repetitive use}

Because RFID is electric data which can be written repetitively, so the tag can be used repetitively.

\subsubsection{Penetrability}

If RFID is covered by the paper, wood, plastics or non-metal or non-transparent materials, it can communicate through these materials except for the irons or other metals.

\subsubsection{Big memory capacity of data}

The data capacity will be extended with the development of the memory scale, and the quality of the material carried by the goods is larger, the requirement of the capacity for the volume label also increase, and RDID will not be restrained.

\subsubsection{System safety}

The system stores the data from the central computer to the work piece which will largely enhance the safety of the system.

\subsubsection{Data safety}

The checkout method or the cycle redundancy checkout method will be used to ensure the data veracity stored in the radio frequency tag.

\section{Total design and work principle of the monitoring system}

\subsection{System design}

Except for fulfilling the function requirements, the design project should fully consider the stability, reliability, anti-jamming ability, fault-tolerance ability and abnormity protection, so the total design of the system should be embodied in following aspects. 
(1) The design could effectively identify and monitor underground personnel, and realize the humanization, informationization and automatization.

(2) The design could effectively offer personnel's passing in and out, attendance and monitoring and relative management information, and once the accident happens, the design could ensure the normal implementation of the rescue.

(3) The design of the system could possess the natures of safety, maintenance and expansion.

(4) The design could effectively test the concentration of harmful gases and quickly react.

\subsection{System work principle}

The underground station equipment emits the encrypted information to the exterior by the antenna and forms certain signal emission region. The RFID cards carried by the workers will be activated when they enter into the emission region, and the information carried with the encrypted objective identification are emitted by the emission module, and the substation equipment will receive these signals and abstract the objective identification code, and transmit the code to the ground computer system to complete the function of the system. When the concentration of the harmful gases achieves certain value, the gas sensor of the substation equipment transmits the information to the A/D converter, and after the information are converted, they are transmitted to the monitoring network to realize the test and monitoring.

\section{Composing and structure of the system}

The safety monitoring system includes the ground part and the underground part (seen in Figure 2).

The products in the system adopt the sap hanging design (Tan, 2007) to ensure the normally and continually running of the system in the severe environment and offer power supply for 1 3 hours after power-down.

The underground part and the ground part adopt the RS-485 serial interface standard communication and the RS-232 standard serial interaction to connect with the transmission network. The data conversion interface is mainly composed by the power supply board, signal conversion board and safety bolt, and it is placed on the mouth of the mine, and it is mainly used to ensure the information exchange between the underground monitoring points and the ground monitoring center, so the system needs not the RFID and A/D converter. Figure 3 is the exchange of the communication signals between the ground and the underground.

\subsection{Underground part}

The underground part is the core part of the monitoring system, and it includes the fieldbus and a series of substations which are used to monitor and control the personnel. The fieldbus is the network which connects the substation and transmits information. Some substations are used to position the underground workers and equipments real time. The positioning mode of the personnel and equipments is the signal strength monitoring method, and when the E-tag carried by one worker is near certain positioning station, the signals acquired by the station are strong, so we can judge the position of the tag, and when the E-tag is in the superposition region of two stations, the signal strengths of two station will be different, and we can judge the position of the tag by comparing the strengths of the signals. Other substations are mainly composed by sensor, A/D converter, micro-controller and RFID module, and they can monitor the harmful gases real time and acquire the transmission of the information, and when the concentration of the harmful gases exceeds the safety value, the ground work platform and the underground fixed monitor point emit the alarm simultaneously. The substation in the underground part adopts the wire communication, the serial interface and the RS-232 serial data interface standard, and the bus transmission and the serial interface are used for the future expansion of the system function.

\subsection{Ground part}

The ground part mainly includes the management operation system, and it is the base of the whole safety monitoring system, and its main functions are to complete the information acquirement, real-time processing and storage of the check point. The information from the underground include not only the concentration of the harmful gases, but the information about the underground personnel and equipment, and these large-size data are all stored in the database through compression as the base of the monitoring and controlling.

\section{Conclusions}

In this article, we applied the RFID technology in the coal mining management, and established an underground safety monitoring system with integrality, real-time and flexibility, which could automatically alarm when dangers happened and enhanced the search and rescue efficiency. The system could manage the harm-gas concentration, planning arrangement of workers, access control for the works passing in and out laneway, distribution of laneway personnel and workers' data, realize the informationization and visualization of the underground management, and largely enhance the mining production management level and the work safety level of the mine.

The safety monitoring system is based on the safe production of the mine, and the module of RFID is the major 
equipment, and the wire communication network is the ligament, and the monitoring central PC computer is the central computer management system. The system could improve the safety mechanism for the miners and reduce the cost of the management, and the technology will be the new trend of the mining safe production monitoring.

\section{References}

Guo, Yanli. (2005). Mine Application Example of South Africa Mining Industry. Automatic Identification Technology \& Application. No. 5.

Klaus Finkenzeller. (2003). RFID Handbook: Fundamentals and Applications in Contactless Smart Cards and Identification (second Edition). John wiley \& Sons, 2003.

Liu, Lili \& Yao, Meng. (2004). Coal Mine Security Intelligent Control System Based on RFID. Global Electronics China. No.9. P.22-25.

Nadeem Raza, Viv Bradshaw, Matthew Hague, et al. (1999). Application of Technology. The Institution of Electrical Engineer, 1999.

Qiu, Like. (2007). The Principle of Staff Positioning System for Those Working in Underground Coalmine and Its Application in Xinglongzhuang Coalmine. Coal Mine Modernization. No.1. P.28-29.

Simson Garfinekel, Beth Rosenberg. (2005). RFID, Applications, Security and Privacy. Addison-wesley, 2005.

Tan, Min \& Liu, Yu. (2007). RFID Technical System Engineering and Application Directory. Beijing: China Machine Press. April of 2007.

Zhou, Xiaoguang \& Wang, Xiaohua. (2006). Technical Principle and Application Example of RFID. Beijing: Posts \& Telecom Press. Dec. of 2006.

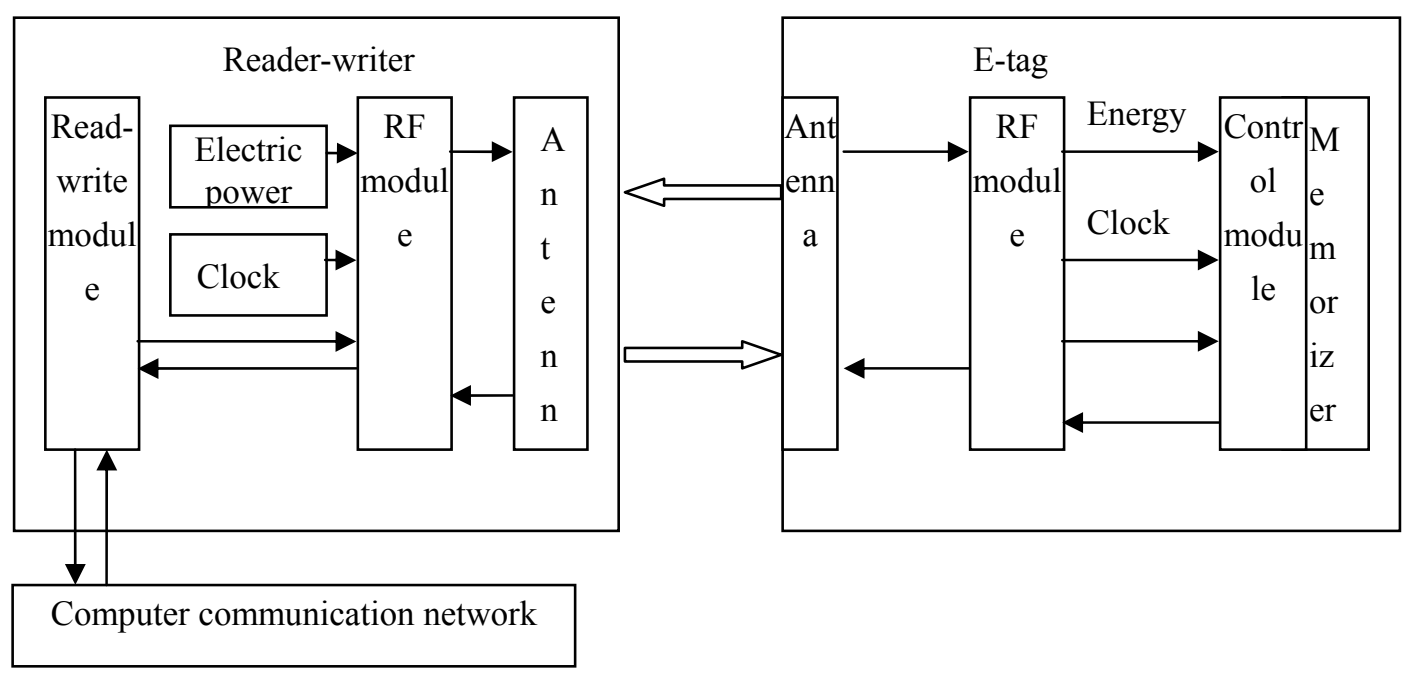

Figure 1. Structure of RIDF System 


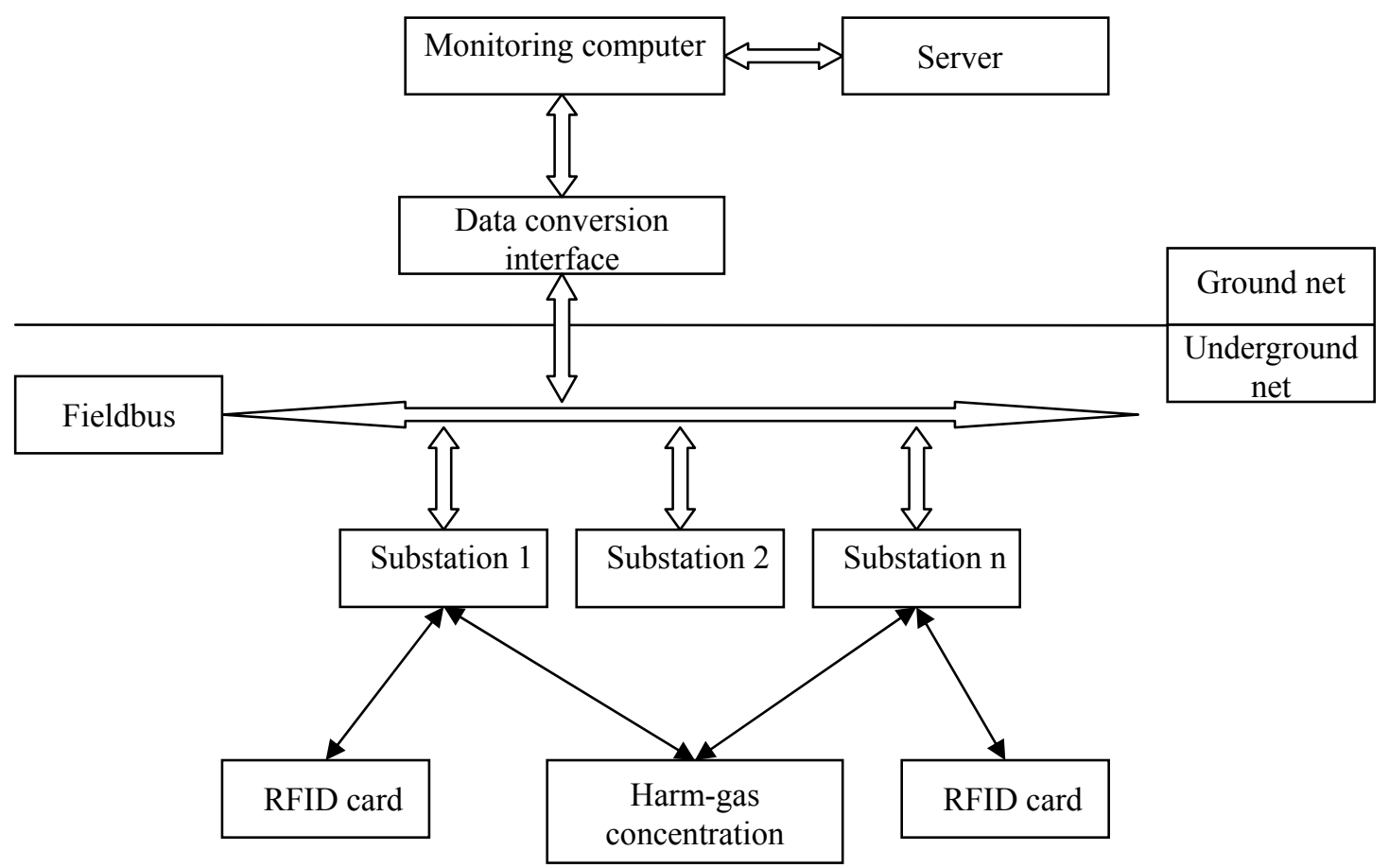

Figure 2. Structure of Safety Monitoring System

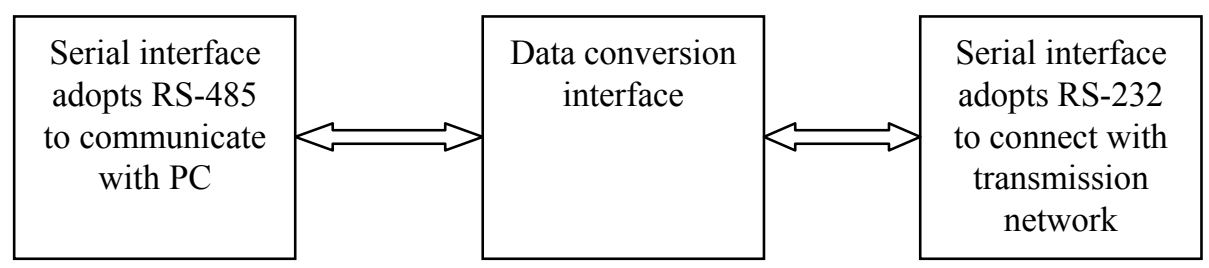

Figure 3. Communication Singles Exchange between Ground and Underground

\begin{tabular}{|c|c|}
\hline \multirow{7}{*}{$\begin{array}{c}\text { Monitoring } \\
\text { computer }\end{array}$} & $\begin{array}{c}\text { Underground personnel positioning and orientation } \\
\text { dynamically display subsystem }\end{array}$ \\
\hline & Attendance subsystem \\
\hline & Mine entrance data acquirement subsystem \\
\hline & Ground/underground alarm subsystem \\
\hline & Harm-gas concentration monitoring subsystem \\
\hline & Communication subsystem \\
\hline & Equipment dispatching subsystem \\
\hline
\end{tabular}

Figure 4. PC Management System 